\title{
The Application of User Innovation and Knowledge Commons Governance to Mental Health Intervention
}

\author{
Glenn Saxe and Mary Acri
}

\section{INTRODUCTION}

User innovation has been applied in many fields (von Hippel 2005), including those related to health care. This chapter describes what is, to our knowledge, the first application of user innovation to the mental health field. We apply user innovation and knowledge commons governance to a mental health problem of considerable importance: child traumatic stress. As we detail, user innovation provides a unique opportunity to develop and adapt interventions that meet the needs of children with traumatic stress and their families. Knowledge commons governance provides a way to share, vet, and improve these user innovations. This approach provides a solution to a critical problem related to the delivery of effective interventions in the mental health field, where the development of effective treatments often is impeded by the inflexibility of evidence-based treatments (Saxe and Acri 2016). First, we describe the problem of child traumatic stress and the imperative to provide effective treatments for children who suffer from it. Second, we detail the problem within the mental health field about adapting interventions so that they meet the needs of individuals with mental health problems and can be delivered in a variety of typical care settings. Third, we describe how we encourage user innovation and harness it in a knowledge commons by creating an intervention model for traumatized children that is flexible enough to address their needs in a variety of typical care settings and by providing infrastructure for sharing and vetting the innovations made by users in adapting the model to their particular circumstances. This intervention model is called Trauma Systems Therapy. It is currently disseminated in 14 states and has been adapted to work in a wide variety of service settings via the process of user

Glenn Saxe is the Arnold Simon Professor and Chair of the Department of Child and Adolescent Psychiatry at New York University School of Medicine and the Director of the Child Study Center at NYU Langone Medical Center, New York, New York. Mary Acri is Research Assistant Professor, Department of Child and Adolescent Psychiatry, New York University School of Medicine, New York, New York. 
innovation. These adaptations have been shared and vetted using a knowledge commons approach.

\subsection{TRAUMA AND CHILD TRAUMATIC STRESS}

Sadly, traumatic adversity has been a part of the human condition over the course of human history, and millions of individuals annually confront traumatic adversity in the form of wars, political violence, interpersonal violence, child maltreatment, community violence, injuries, and life-threatening medical illnesses in the United States and around the globe. The impact of trauma on children is revealed through the following facts:

- From the general public in the United States, 52.1 percent of adults have reported having at least one adverse childhood experience; 22 percent reported sexual abuse and 10.8 percent had experienced physical abuse (Felitti et al. 1998).

- In 2012, 4.5 children died every day across the United States due to abuse and neglect (US Department of Health and Human Services [HHS], Administration for Children and Families, Administration on Children, Youth and Families [ACYF], Children's Bureau 2013).

- Also in 2012, 3.2 million children received a child protective services response (HHS, ACYF, Children's Bureau 2013).

- In a nationally representative sample, 6 o percent of children were exposed to violence, and 46 percent were assaulted within the past year (Finkelhor, Turner, Ormrod, Hamby, and Kracke 2009).

- Among New York City families receiving child welfare services, 92 percent of children have been exposed to at least one traumatic event, and 86 percent have experienced multiple traumatic events. Among mothers, 92 percent have experienced a traumatic event, and 19 percent have experienced five or more types of traumatic events (Chemtob, Griffing, Tullberg, Roberts, and Ellis 2010).

- Childhood exposure to adverse experiences increases the risk for alcoholism, suicide attempts, depression, drug abuse, obesity, heart disease, and cancer (Felitti et al. 1998).

The most common response to traumatic exposure - post-traumatic stress disorder (PTSD) - involves responses to a traumatic event that include the following symptom categories (American Psychiatric Association 2013: 5-25):

Re-experiencing: Involves ongoing intense memories of the traumatic event, flashbacks, nightmares, and distress at reminders of the traumatic event.

Avoidance: Involves efforts to avoid thoughts and people, places, and things that are reminders of the trauma. 
Negative cognitions and mood: Involves ongoing negative thoughts and feelings about the trauma such as persistent self-blame, diminished interest in activities, and inability to remember key aspects of the trauma.

Hyperarousal: Involves difficulty staying calm, startle responses, poor sleep, and vigilantly anticipating new traumas.

Obviously, there is a great need to develop effective interventions for traumatized children that will meet their needs and will be scalable in typical care settings. In fact, several interventions have been developed for children with traumatic stress that have demonstrated efficacy and effectiveness in clinical trials. A problem is that few have been able to be widely scaled in typical care settings with similar levels of fidelity or outcomes.

\subsection{THE PROBLEM WITH EVIDENCE-BASED MENTAL HEALTH}

\section{INTERVENTIONS}

The problem with interventions for child traumatic stress is the same for other evidence-based mental health interventions. Most effective treatments do not reach those for whom they were intended (Mitchell 2011; Proctor et al. 2009). Dissemination efforts are slow (Proctor et al. 2009), and evidence-based treatments have been cited as inflexible and difficult to integrate into community clinics (Mitchell 2011). As a result, effective treatments for a host of mental health disorders, which costs taxpayers billions of dollars to study, fail to reach those in need to the detriment of millions of individuals currently experiencing psychiatric problems (Proctor et al. 2009).

A key part of the problem - and one that user innovation is ideally designed to address - relates to the great caution expressed within the mental health field about implementing an evidence-based treatment (EBT) in a way that deviates from the fidelity standard used in the clinical trial from which it gained its evidence base (Moore, Bumbarger, and Cooper 2013). A fidelity standard for a mental health intervention details the specific psychotherapeutic procedures that should be followed by a mental health provider with a person with a mental health problem for the improvement of that mental health problem. For example, a fidelity standard for an intervention such as cognitive behavior therapy for a psychiatric disorder such as depression would involve an assessment of the degree to which the clinician corrected distortions in thoughts (cognitions) about the self (e.g., "I am bad"), the world (e.g., "Everyone would be happy if I were dead"), and the future (e.g., "Nothing will ever change") since these types of thoughts/cognitions are believed to be central features of depression. Accordingly, cognitive behavior therapists are trained to correct such cognitive distortions as an essential part of their intervention approach, and the degree to which the correction of cognitive distortions is observed within cognitive behavior therapy is a primary way of assessing fidelity to this intervention model. Fidelity standards will often include details on the frequency and duration of sessions, the level of training of the clinician, as well as 
many details about how the central components of the intervention are to be delivered (e.g., for cognitive behavior therapy, details on how the cognitive distortions are to be corrected). Such procedures are written in a standardized format - often in great detail so that they can be reliably delivered. The clinical trial that established the evidence base for the intervention employed its defined fidelity standard in the process of achieving the defined outcome.

An intervention's fidelity standard usually contains little information about how the procedures it specifies might be applied to the specific settings in which it would be implemented. Specific circumstances that might affect implementation include the complexity and diversity of a particular settings' clinical population; its providers, supervisors, and administrators; its organizational processes (including finances); and the specific service system in which that organization must operate. This rigidity seriously limits the effectiveness with which evidence-based treatments can be disseminated to those who need them. Successful implementation of an intervention would often necessitate adapting it to specific local circumstances via the development of new or adjusted procedures that would "accompany" the delivery of the given intervention (e.g., delivering the EBT with less frequent sessions, delivering the EBT to broader clinical populations). Under the standard approach to evidence-based treatment, however, such innovations would be considered fidelity violations.

Overly rigid fidelity standards also greatly limit the capacity of an intervention to be adjusted over time, based on experience. When a mental health intervention cannot be changed because any change becomes a fidelity violation (which then violates the evidence-basedness of the intervention), our interventions become fixed in time and the critical process of intervention improvement is stifled. The current standard paradigm for quality improvement in the mental health field, which requires the delivery of treatment in a way that adheres closely to a rigidly defined fidelity standard that was evaluated in the treatment's clinical trial excludes information from users of the treatment - including clinicians, clinic administrators, patients and their families - from the process of intervention development, innovation, and adaptation. We believe, however, that it is exactly the integration of this local information - which is held by users - that is required for an intervention to continue to improve in an evidence-based fashion.

\subsection{WHY USER INNOVATION?}

User innovation - by definition - integrates the needs of users into the process of developing products that will meet their needs. It incorporates their local knowledge of their particular problems and available resources that is not available to those traditionally expected to provide innovations - typically a manufacturer or expert. As described, there is usually no process for integrating users' local information into mental health intervention development, innovation, or adaptation. This is - in 
essence - a problem of the limitations of expertise that is analogous to the limitations of manufacturer knowledge typically explored in user innovation research. Mental health intervention developers may be expert in many areas. They may have a great deal of knowledge about the mental disorder in question and the research literature on interventions for the disorder. They may be experts on the standardization of interventions, the development and implementation of clinical trials, and the use of data from these clinical trials. Intervention developers - of whichever sort - do not have expertise about the specific settings in which their intervention will be implemented, however. Who has this expertise? The answer is the users of the intervention within these specific settings.

User innovation can leverage the expertise and knowledge of users in a variety of settings to expand the range of applicability of a treatment model and to improve it. To create an impactful and scalable mental health intervention for child traumatic stress, we designed our treatment model - Trauma Systems Therapy (TST) - with the user innovation process in mind, allowing us to leverage local user expertise in its development, innovation, and adaptation while preserving the value of the evidence-based approach. User innovation research also demonstrates that, to get the most out of the potential for user innovation, innovations must be shared, vetted, and improved upon by other users in a user community or knowledge commons. Our approach involved various measures aimed at facilitating such a knowledge commons for TST.

\subsection{TRAUMA SYSTEMS THERAPY}

As detailed in our book entitled Trauma Systems Therapy for Children and Teens (Saxe, Ellis, and Brown 2016), TST is both a clinical model for the treatment of children with traumatic stress and an organizational model for the successful implementation of the TST clinical model based on the way organizations work. It takes a defined position on the core clinical problems of traumatic stress in children and a defined position on how organizations can best support and sustain their TST programs. According to TST, traumatic stress occurs when a child is unable to regulate emotional states and in certain moments experiences his or her current environment as extremely threatening even when it is relatively safe. TST specifies interventions that address what is called a "trauma system," which emerges in response to disruption in the natural systemic balance between the developing child and his or her social environment. A trauma system is composed of the following:

1. A traumatized child who experiences survival states in specific definable moments. A survival state occurs when a child experiences her or his environment as threatening to survival when reminded of a past traumatic event. 
2. A social environment and/or system of care that is not able to help the child regulate these survival states.

TST explicitly addresses these two core problem domains. Because the social environment (e.g., family, school, peer group, neighborhood) ordinarily has a core function of helping a child contain emotions or behaviors, we assume that a child's inability to contain emotions or behaviors reflects a diminished capacity of one or more levels of that social environment to help the child. Correspondingly, a child's inability to regulate emotional states presumptively implies some inadequacy in the system of care to help the child contain emotions or behaviors. The following two brief examples illustrate these issues:

1. A ten-year-old boy was repeatedly beaten by his father for reticence to engage in sports, and for his father's opinion about his poor performance on the sports field. The boy experiences severe anxiety prior to physical education class at school and has missed physical education repeatedly by visiting the school nurse for a variety of physical complaints. He experiences severe flashbacks about the assaults during the day and his grades at school have diminished because he cannot focus on his lessons.

2. A 15-year-old girl was sexually assaulted by her mother's boyfriend. She experiences a lot of pressure from her friends and from her mother to talk with and to date male classmates. She experiences severe anxiety and flashbacks about the sexual assault whenever she thinks about dating boys and whenever a male gets too close to her. The pressure from her friends and her mother also induces flashbacks. She has nightmares of the assault every night. Although she has told her mother about the assault, her mother continues be in a relationship with the man who assaulted her.

As can be seen from the brief descriptions of these children, their problems involve both the dysregulation of emotional states related to extreme threat/survival (e.g., severe anxiety, flashbacks, nightmares, avoidance) and problems within their environment for helping and protecting them (e.g., in the boy's case: how the school handles issues of attending physical education class and school performance, at home the ongoing threat from father. In the girl's case: the ongoing pressure from friends and mother to date boys, mother's continuing relationship with man who assaulted her daughter and lack of protection of her daughter).

To address these problems, TST provides a format for integrating interventions often provided within separate care settings, such as psychotherapy, psychopharmacology, and home-based care, and specifies how these modalities of intervention should be practiced. TST also specifies the processes by which mental health organizations should integrate and support the services they provide. These processes include the establishment of a multidisciplinary treatment team to collaborate on the assessment and treatment of children receiving TST, and the training and supervision process for ensuring TST is delivered with sufficient fidelity. 


\subsection{USER INNOVATION, KNOWLEDGE COMMONS GOVERNANCE AND TST}

User innovation and knowledge commons governance were facilitated within the development and dissemination of TST via five commitments made and supported by the chief developers of TST:

1. A commitment to the public nature of intellectual property related to TST. One obvious barrier to innovation is a concern that one will trespass on ownership claims by others in doing so. Indeed, developers of mental health interventions commonly do make ownership claims. We took a different approach. The TST book makes the waiver of our claim to intellectual property explicit. The waiver is accompanied by an invitation for users to feel free to adapt TST to their needs and to share their experiences with us, and with others. This commitment is a necessary step to facilitate user innovation by providing openness to change TST without concern for violating intellectual property laws. This commitment is also a necessary step to facilitate an informational commons by permitting complete openness to the sharing of information related to TST:

TST is public property. It is designed to be used and is owned by its users. If you use TST, we consider you part of our innovation community and hope that you will use your skill and creativity to adapt it to best suit your needs, and more important, to the needs of the children and families you serve. If you make a useful adaptation to TST based on what will work in your organization, that is great and we hope you will share your innovation with us. It is our sincere hope that these innovations will continually work to create an ever-improved TST, and a model that users can feel they own (Saxe et al. 2016).

2. A commitment to a minimal fidelity standard. In developing our fidelity standard, we have attempted to limit its restrictions to elements that we believe should minimally be in place for TST to be properly delivered, based on our experience - as user innovators ourselves - in delivering TST in many settings, over many years. Such minimal fidelity processes are conceptually similar to the goals of user "toolkits" in manufacturing fields (von Hippel 2001). A mental health intervention's fidelity standard is important. If it is not sufficiently well defined, then intervention cannot be specific enough to be effective. If it is overdefined then it is prohibitively difficult for users to fit the intervention to their local circumstances and needs. We believe that over-definition is a very big problem within the mental health interventions field. By developing a minimal fidelity standard that includes only the elements necessary to give us confidence that the treatment will be deployed appropriately, we aimed to encourage users to adapt TST to their needs in any fashion they want over and above the established minimal fidelity standard. (In truth, since we have not claimed intellectual property, nothing precludes a user from changing TST in a way that violates even the established minimal fidelity standard. In our experience, users have not 
sought to do this.) The TST minimal fidelity standard thus becomes the platform on top of which all innovation occurs for the TST model.

3. A commitment to a community of innovators. User innovations are most effective when they are shared within a user community that can vet, improve upon, and disseminate them. There are barriers to such sharing, however. In the mental health arena, these barriers include concerns about possible ownership claims or issues of deviating from the fidelity standard and the time and other resources that such sharing may require of busy and resource-strapped local mental health providers. We have taken specific steps to overcome these barriers. Our initial commitment to encouraging users to innovate above the TST minimal fidelity standard removes potential concerns that sharing will attract negative reactions based on ownership claims. We have also created infrastructure to help overcome resource-based and other barriers to sharing. TST implementers are invited to join what we have called the TST Innovation Community to share their TST innovations and to learn from one another. The TST training team is based at the NYU Child Study Center and manages monthly conference calls for members of the TST Innovation Community to facilitate this sharing. A webbased portal located on the TST website is also available so that members can download the most current, official versions of all TST tools/forms, upload tools/ forms created by member sites (with approval by the model developers) to be made available for all members to download and use, and communicate with one another via an interactive blog. Our continued involvement in supporting the TST Innovation Community makes it a more valuable resource and thus encourages users to join. It also helps assuage concerns that we, as the initial developers, or potential users might have about quality control. In this respect, our system functions much like most widely used open source software projects, which permit users to make and use their own modified versions of the code but maintain official releases of the software that are vetted and controlled by some coordinating body, usually including the initial developer of the code.

4. A commitment to the scientific process in standardizing and evaluating innovations. One of the reasons for over-specifying a fidelity standard for a mental health treatment is a concern by the initial developers that deviations from the standard will have adverse impacts on treatment effectiveness. Indeed, most users of TST are community-based providers who do not have the time, resources, or expertise to standardize or evaluate mental health intervention innovations. The standard approach to disseminating a treatment model provides no mechanism for addressing this concern. By adopting a community-based knowledge commons approach, we maintain contact with the TST user community and are in a good position to help users ensure that their innovations are effective. To that end, our NYU team provides technical assistance to user organizations so as to standardize and evaluate the utility of the innovations that they share with us. Thus, our process not only maintains a commitment to evidence-based 
treatment based on our initial trials, but it also permits the continuing evaluation of the treatment's effectiveness as it is adapted for use in a range of local circumstances.

5. A commitment to the continual improvement of TST over time. Our vision is for TST to develop as a platform for innovation in service delivery for traumatized children and families such that it grows with an ever-expanding set of standardized innovations developed by users to meet their specific needs and then implemented by other users with similar needs. In this way we have always seen the development of TST as a never-ending story of innovation with the continuing integration of new standardized and evaluated innovations based on their demonstrated utility. In our book we use the example of the release of the iPod by Apple in October 2001 to illustrate why this is so important.

TST has become a never-ending story based on a community of users. If we had stopped when we completed our manual in 2001, or when our first outcome study was published in 2005, or when our first book was released in 2006, it would have been like stopping the development of the iPod after its release on October 23, 2001 (we are not claiming that TST is as groundbreaking as the iPod - that's up to you to determine). We believe this tendency to stop improving a model has become a big problem in the field of psychotherapy development. A psychotherapy model gets developed and evaluated, a clinical trial gets published about the model's efficacy, and the development of the model then largely stops - because any change to the model becomes a fidelity violation and means that an implementation of the model, with this change, cannot be supported by the evidence base of the clinical trial. We are creating a field of 2001 iPods! (Saxe et al. 2016: 353-64).

\subsection{RESULTS OF OUR USER INNOVATION AND KNOWLEDGE COMMONS-BASED APPROACH}

Our user innovation and knowledge commons-based approach has been extremely successful in disseminating our treatment model. TST is now used within mental health agencies in 14 states and three nations. Moreover, establishing and maintaining our commitments to user innovation and knowledge commons governance as detailed earlier has led to the integration of many user innovations into TST, creating an intervention model that is much more flexible to responding to the needs of users than we could have possibly achieved without our community of users. Given the opportunity to innovate, users have adapted the model to settings and clinical problems that the developers never imagined when we set out to design TST. Here we briefly describe some of these innovations. For those who are interested, more details are given in our book. 
None of the settings described here were considered in the initial design of TST. We designed TST at first as an outpatient mental health program within a hospital that served inner-city children and families (Boston Medical Center). It thus was designed as a treatment model for children with traumatic stress who receive care in outpatient mental health settings in urban environments. We never imagined that TST would turn out to be useful for programs outside this context. Providers in such programs imagined this use for us.

1. Residential Care Settings

What it is: Children in residential care settings cannot live with their families for periods of time that range from weeks to months. Usually, a child will be admitted to a residential care program because he or she has a significant mental health problem and his or her behavior poses a risk to the child, or others. Often the child has significant family problems that have contributed to his or her difficulties.

Why TST was thought to be a good fit: A large proportion of children in residential settings have mental health problems related to trauma and the dangerous behaviors are related to shifts to survival-laden emotional states described previously. Often the shift to these states is related to family problems. Once these children arrive in residential programs, shifts to a survival state occur within the programs and the child's providers want to do a better job helping the child regulate emotion. The trauma system of a child's dysregulation of emotional states within the social environment of the residential care setting and the child's home setting was thought to be an excellent fit for TST.

How TST was adapted to fit: Tools were created to assess survival states within residential settings:

- Residential care settings are managed by both clinical and nonclinical staff. Processes to integrate nonclinical staff were created.

- The TST organizational approach was adapted for the needs of administrators of residential care settings to address all services that interact with the child (e.g., psychology, social work, psychiatry, school, direct care, recreation).

- Residential care settings are usually affiliated with schools located on the site of the residential program. Processes to integrate schools were created.

- Many residential programs struggle with physically restraining children. Tools to help programs intervene without restraint were created.

\section{Foster Care Settings}

What it is: Children in foster care have been removed from their families because the child welfare authorities have determined sufficient maltreatment has occurred to mandate such removal and placement. 
Why TST was thought to be a good fit: Traumatic stress reactions are common among children in foster care. The ongoing maltreatment, the removal from family, and the placement with a family that is usually unknown to the child set the stage for ongoing traumatic stress, survival-in-the-moment reactions. Agencies seeking TST for the foster care system have typically wanted a trauma-informed program that could prevent foster care placement disruptions and facilitate reunification with families, when appropriate.

How TST was adapted to fit:

- Teams are created that include mental health clinical providers and foster care case workers. Caseworkers and foster care staff provide the home-based component of TST intervention.

- Foster care placement disruptions are often driven by the child's repetitive shifts to survival in the moment. Foster families can be frustrated (and frightened) by the seemingly "out-of-the-blue" nature of the child's survival states. Accordingly, information about these states can be very helpful for foster families. A foster parent with a child who, for example, shifts to survival in the moment with specific struggles around food and mealtimes could be helped to understand how these struggles could be minimized and survival in the moment prevented.

- Placement decisions are informed by an understanding of a child's vulnerabilities defined by his or her propensity to shift into survival states. Some foster parents may have greater capacity than others to address a child's difficulties. In preparation for the placement of any foster child, the foster family should understand the child's specific vulnerabilities, as described, and needs to be supported by the foster care agency to manage them.

- Reunification decisions are informed by the understanding of the child's vulnerabilities defined by his or her propensity to shift into survival states. Before reunification is considered, the role of specific family members in the child's problem is examined with a view to whether there has been sufficient improvement. If a child's survival states typically involve suicidal or violent behavior, and these states have repeatedly been driven by specific interactions with family members, it is not surprising that they would recur upon reunification if there has been no effective intervention with those family members.

3. TST with Refugee Populations:

What it is: Refugee populations have a high prevalence of traumatic stress related to war and political violence in their nation of origin and the experience of displacement and resettlement. The cultural stressors related to resettlement present particular difficulties within the family context. 
Why TST was thought to be a good fit: Agencies seeking TST for refugee populations typically are looking for trauma-informed programs that address the social environment, integrate the cultural context, and connect to the wider services system.

How TST was adapted to fit:

- The refugee child's home environment is shaped not only by the particular family members but also the larger culture of origin that influences the families' goals, behavior, and way of relating to the world beyond. Mental health clinicians typically cannot understand and appreciate the cultural nuances of cultures they are not a part of; cultural brokers, or members from the refugee community who have a deep understanding of their own culture, the resettlement culture, and mental health service systems culture, are integral members of the team. Cultural brokers can help with language translation and, perhaps more importantly, the translation of cultural meanings that relate to such factors as trauma, emotional disorders, and mental health intervention. The identified cultural broker is an integral member of the TST team.

- Broader community outreach provides a foundation from which the specific TST program can operate. Refugee communities can have great wariness about those in authority, and mental health providers are seen as those in authority. The engagement of the community, and a process of co-learning about both the community's primary concerns and the way trauma may be related helps engage whole communities in overcoming stigma and supporting their children in receiving the services they need.

- The inclusion of cultural and religious practices that children and family members find comforting and helpful can be important. The identification of these practices is assessed and integrated as strengths during the treatment planning and engagement process. They are included in the emotional regulation interventions. Partnering with religious leaders can be helpful here.

- The stigma of mental health problems and intervention can present large barriers to engagement with some refugee communities. Accordingly, it can be helpful to base TST refugee programs in less stigmatized settings such as within primary care or schools. In addition, non-stigmatized skill-building groups offered in a school setting allow families and children to begin engagement with TST ideas and team members in a way that does not single their child out as in need of services, but instead acknowledges the ubiquity of cultural stress and supports all refugee children's adjustment to a new cultural setting. 
4. TST with Substance Abusing Adolescents:

What it is: The rates of trauma and victimization histories are high among adolescents presenting for substance abuse treatment. Childhood trauma exposure increases risk for later substance use, criminal activity, anxiety disorders, and so on. Youth who exhibit comorbid substance abuse and post-traumatic stress problems show greater clinical severity, increased functional impairment, and greater involvement with multiple service systems when compared to youth with only one of these conditions.

Why TST was thought to be a good fit: Agencies seeking TST for substanceabusing populations typically are looking for a treatment approach that can address how substance abuse may be related to traumatic stress reactions and that integrates the way the youth's social environment may contribute to these problems, across multiple services systems.

How TST was adapted to fit:

- Substance- abusing behavior is considered as an emotional regulation process related to survival states. Moment-by-moment analyses gather information about the patterns of these shifting states so that the contributors to the adolescent's substance abuse can best be understood.

- As the youth's substance abuse deepens, stimuli related to trauma diminish in importance as a result of the process of chemical dependency. Environmental cues related to substance abuse grow in importance. Trauma-related stimuli may continue to be contributors throughout but their relative importance diminishes. Accordingly, TST may be more effective earlier in the chemical dependency process than later.

- Psycho-education about substance abuse and its interaction with symptoms of traumatic stress are integrated in the treatment.

- Behavioral management techniques are particularly emphasized with substance-abusing adolescents. Behavior management strategies integrated into TST include increased substance abuse monitoring and appropriate limit setting, particularly around drug use and high-risk behaviors.

- The addition of substance abuse treatment strategies such as parent-teen communication skills, recognizing and planning for substance abuse cues or trigger situations, substance abuse cravings, cognitive and interpersonal problem-solving techniques, and other relapse-prevention techniques.

5. TST in Schools:

What it is: Children with traumatic stress can have particular problems in schools, and schools based in communities with high rates of trauma can have difficulties with a host of problems related to traumatic stress. Children who 
experience survival-in-the moment in schools can be highly disruptive in the classroom, and then they get suspended. These children can become quite avoidant of school and may have high rates of absenteeism. Children with traumatic stress may also be quietly dissociative in class and not learn at their intellectual capacity. Children with survival responses frequently are seen as discipline problems, unmotivated, or as children who are angry and over-reactive. This common, but inaccurate, interpretation of survival states by teachers often leads them to respond in ways that are not helpful and may even exacerbate the problem. A child who has been suspended for disruptive behavior, or who has a poor school attendance history, or who does not pay attention in class, is a child at high risk of school failure and/or dropping out.

Why TST was thought to be a good fit: Schools seeking TST typically are looking for an integrated treatment approach that can help with the children they are most worried about for reasons described earlier. They seek a program that can fully engage the social environment of the school and the teachers and other staff members of the school to better understand the school-related problems of traumatized children.

How TST was adapted to fit:

- The TST program in the school integrates within the school culture and performs a vital consultative role helping teachers and other school staff to see how traumatic stress can influence learning at school. Trainings should be offered by the TST team and direct classroom observations conducted to provide consultation to classrooms with particular difficulty.

- The organization plan within the school should be drafted with a leadership team that integrates representation from all specialties that will be involved in the care of children. This includes special education, physical education, security, and regular classroom teachers, as well as representation from the principal's office.

- There should be an evaluation plan that directly addresses the concern of the school (particularly the principal's office). Such a plan should track key educational indicators such as absenteeism, classroom disruptions, critical incidents, and school performance.

- The assessment of children should include classroom observation, particularly if there is concern about behavior in the classroom.

- Most schools do not have dedicated teams of mental health providers (although many have a small capacity for learning and behavioral consultation). Schools will typically refer to local mental health agencies for the children who cause concern, but that work then is usually quite disconnected from the school and does not address the social environment of the school. Successful programs have formed partnerships with local mental health agencies so that clinicians from those agencies join the TST team 
and conduct the mental health intervention integrated with the work of the team and with fidelity to TST. Such providers, obviously, add to the mental health capacity of the TST program in the school. The interagency agreement between the school and the mental health clinic allows the mental health clinicians to "bill" for services, as usual, under the mental health clinic's license. This can be financially advantageous to the mental health clinic as providing intervention at the child's school reduces noshow rates.

- The child's family is integrated into care. If a child needs safety-focused treatment, there is a clear problem at home that needs to be addressed. Mental health agencies that provide home-based care for children and families with acute need should be integrated to the program and engaged through interagency agreement as described earlier. If a child needs safetyfocused treatment, he or she may well be in danger at home. The child may also be highly disruptive in class. In such a situation, the situation will not improve without the integrated home-based services that are part of treatment. These are the children who are usually thought to be too difficult to handle in school. They get psychiatrically hospitalized and then lose their school placement.

- The TST team evaluates children's individualized education plans (IEP) for their suitability related to the impact of traumatic stress on the child's learning at school. TST providers attend the IEP meetings of the children they work with. There should be an agreement, reflected in the programs organizational plan, about how the situation will be handled if the TST team and the school's educational program disagree about IEPs.

As seen by these examples, an ever-broadening community of users and innovators adapted TST to their needs and became considerably, and increasingly, more useful than it was when first launched; it is now available to any organization that wants to use it. And it has been specifically adapted to a wide range of settings and for a diverse range of populations of traumatized children.

\section{CONCLUSION}

Undoubtedly, user innovation and knowledge commons governance have far-reaching potential to improve evidence-based treatments in the mental health field. To adopt this methodological approach, and the theory underlying it, however, developers will have to recognize the limitations of the standard paradigm, be willing to relinquish their "ownership" rights, and be open to allowing users to innovate in adapting a given treatment across settings and populations. Developers will also have to be ready to invest time and resources in maintaining a vibrant user community knowledge commons. While there are some costs and risks to adopting this 
approach, we believe the payoffs from wider employment of the user innovator community paradigm - to the field and to the well-being of children and adolescents with mental health problems - would be considerable.

\section{REFERENCES}

American Psychiatric Association, 2013. Diagnostic and Statistical Manual of Mental Disorders. 5th ed. Arlington, VA: American Psychiatric Publishing.

Chemtob, C. M., Griffing, S., Tullberg, E., Roberts, E., and Ellis, P., 2010. Screening for trauma exposure and posttraumatic stress disorder and depression symptoms among mothers receiving child welfare preventive services. Child Welfare 9o(6):109.

Felitti, V. J., Anda, R. F., Nordenberg, D., Williamson, D. F., Spitz, A. M., Edwards, V. et al., 1998. Relationship of childhood abuse and household dysfunction to many of the leading causes of death in adults. The Adverse Childhood Experiences (ACE) Study. American Journal of Preventative Medicin 14(4):245.

Finkelhor, D., Turner, H., Ormrod, R., Hamby, S., and Kracke, K., 2009. Children's Exposure to Violence: A Comprehensive National Survey. Washington, DC: US Department of Justice, Office of Justice Programs, Office of Juvenile Justice and Delinquency Prevention.

Mitchell, P. F., 2011. Evidence-based practice in real-world services for young people with complex needs: New opportunities suggested by recent implementation science. Children and Youth Services Review 33:207.

Moore, J. E., Bumbarger, B. K., and Cooper, B. R., 2013. Examining adaptations of evidence-based programs in natural contexts. The Journal of Primary Prevention 34 (3):147.

Proctor, E. K., Landsverk, J., Aarons, G., Chambers, D., Glisson, C., and Mittman, B., 2009. Implementation research in mental health services: An emerging science with conceptual, methodological, and training challenges. Administration and Policy in Mental Health and Mental Health Services Research 36(1):24.

Saxe, G. N., Ellis, B. H., and Brown A. B., 2016. Trauma Systems Therapy for Children and Teens. 2nd ed. New York: Guilford Press.

Saxe, G. N. and Acri, M., 2016. Democratizing implementation and innovation in mental health care. Administration and Policy in Mental Health and Mental Health Services Research. doi: 10.1007/s10488-015-0714-1.

US Department of Health and Human Services, Administration for Children and Families, Child maltreatment. www.acf.hhs.gov/programs/cb/research-data-tech nology/statistics-research/child-maltreatment. Downloaded Nov. 10, 2015. von Hippel, E., 2001. Perspective: User toolkits for innovation. Journal of Product Innovation Management 18(4):247.

von Hippel, E., 2005. Democratizing Innovation. Cambridge, MA: MIT Press. 